\title{
Effect of caffeine on functions of cooling-stored ram sperm in vitro
}

\author{
Eliška Špaleková ${ }^{1}$, Alexander V. Makarevich ${ }^{1}$, Elena Kubovičová1, Alexander Ostró ${ }^{3}$, \\ Peter Chrenek ${ }^{1,2}$ \\ ${ }^{1}$ Animal Production Research Centre Nitra, Slovak Republic \\ ${ }^{2}$ Slovak University of Agriculture in Nitra, Department of Biochemistry and Biotechnology, Slovak Republic \\ ${ }^{3}$ P. J. Safárik University in Košice, Faculty of Medicine, Slovak Republic \\ Received March 18, 2013 \\ Accepted December 4, 2013
}

\begin{abstract}
Caffeine is a well-known sperm motility stimulator, however, its effects on cooling-stored ram semen are unknown. The aim of the study was to examine the effect of caffeine on selected motility and viability indices of cooling-stored ram spermatozoa. Sperm ejaculates from 4 rams were diluted $(1: 3)$ in a Triladyl extender. Samples were stored for $96 \mathrm{~h}$ at $4-5{ }^{\circ} \mathrm{C}$ in two sets. In the first set used for motility analysis, caffeine at concentrations of 1,2 or $4 \mathrm{mmol} \cdot \mathrm{l}^{-1}$ was added to sperm aliquots on the day of analysis. In the second set used for viability assay, caffeine at the same concentrations $\left(1,2\right.$ or $4 \mathrm{mmol}^{-1} \mathrm{l}^{-1}$ ) was added at the beginning of storage. Control was left without caffeine addition. Sperm motility was analyzed at 0,24, 48 and $72 \mathrm{~h}$ of cooling-storage. Viability assays were done after $72-96 \mathrm{~h}$ of cooling-storage. Caffeine significantly $(P<0.05)$ increased sperm motility and progressive movement and maintained this value for $72 \mathrm{~h}$. Caffeine at the dose of $2 \mathrm{mmol}^{-1} \mathrm{l}^{-1}$ and $4 \mathrm{mmol} \cdot \mathrm{l}^{-1}$ significantly $(P<0.05)$ reduced the proportion of dead/necrotic sperm detected by propidium iodide and proportion of apoptotic sperm detected by Yo-Pro-1, respectively. No effect of caffeine on plasma membrane integrity was noted. Proportion of sperm with membrane destabilization (annexin V-Fluos) was reduced by caffeine given at 1 and $4 \mathrm{mmol} \cdot \mathrm{l}^{-1}$ compared to control. Our study for the first time demonstrates that caffeine maintains motility and viability of cooling-stored ram sperm for longer time compared to control.
\end{abstract}

Methylxanthines, membrane integrity, membrane stability, apoptosis, motility

Progress in the use of artificial insemination is related to search for substances with the potential ability to improve the fertilizing capacity of spermatozoa. Caffeine is a cyclic nucleotide phosphodiesterase inhibitor which stimulates sperm motility, fructolysis, and respiration and also increases cyclic adenosine monophosphate (cAMP) titres (Fraser and Monks 1990). Positive correlation between the intracellular concentration of cAMP and the rates of progressive motility, fructose utilization, and protein synthesis by ram spermatozoa was observed (Salem et al. 1992). The effects of caffeine are concentrationdependent and species-specific. Studies on bulls revealed that $4 \mathrm{mmol} \cdot \mathrm{l}^{-1}$ of caffeine is an ideal concentration for semen fortification to improve the preservability and postthaw sperm motility (Singh and Raina 2000). At higher concentrations (10 $\left.\mathrm{mmol} \cdot \mathrm{l}^{-1}\right)$ caffeine induces an increase in intracellular calcium and the immediate hyperactivation of incubated ram sperm (Colas et al. 2010). Compared to fresh semen, cooled semen is more predisposed to decrease in sperm motility and higher incidence of morphological alterations, which may reduce sperm fertility and increase embryonic loses (A is en et al. 2002; Gil et al. 2003). The data on caffeine effect on frozen-thawed semen are available; however, there are no reports on the effects of caffeine on cooling-stored ram semen evaluated in relation to indices of sperm viability and motility.

The aim of the study was to examine effect of caffeine on selected variables of coolingstored ram semen. Sperm motility (CASA) and selected characteristic of sperm viability (sperm membrane integrity and membrane stability, sperm apoptosis) following liquid storage of sperm under hypothermic conditions were evaluated. 


\section{Semen collection}

\section{Materials and Methods}

All the experiments were carried out with fresh ram spermatozoa. The semen was collected from 2 EastFriesian and 2 Lacaune rams using an artificial vagina. Ejaculates from all rams were pooled together to make heterospermia and diluted in a Triladyl (1:3) extender (Minitüb, Tiefenbach, Germany) to a final concentration of $1 \times 10^{6} \cdot \mathrm{ml}^{-1}$. Semen was cooled down to $4{ }^{\circ} \mathrm{C}$ and transported to the laboratory. Samples were stored at $4-5^{\circ} \mathrm{C}$ for $96 \mathrm{~h}$ in 2 sets. In the first set used for motility analysis (CASA), caffeine at concentrations of 1,2 or $4 \mathrm{mmol} \cdot 1^{-1}$ was added to sperm aliquots on the day of analysis. In the second set used for viability assay, caffeine at the same concentration $\left(1,2\right.$ or $\left.4 \mathrm{mmol} \cdot \mathrm{l}^{-1}\right)$ was added at the beginning of storage. Control was without caffeine addition.

\section{Analysis of sperm motility}

We analyzed motility variables of spermatozoa at $0,24,48$ and $72 \mathrm{~h}$ of cooling storage, using computer assisted semen analysis (CASA) - Hamilton Thorn motility analyzer (version 7). Sperm samples were diluted in saline containing $1 \%$ of foetal calf serum (saline-FCS). Each sample was analyzed at the time intervals of $0,0.5 \mathrm{~h}$ or 2 $\mathrm{h}$ following caffeine addition. Between these time points the samples were incubated at $37^{\circ} \mathrm{C}$. We analyzed the effect of various concentrations of caffeine on ram sperm total motility, progressive movement (PM), and other sperm characteristics, i.e. curvilinear velocity (VCL), straight line velocity (VSL), average path velocity (VAP), amplitude of lateral head displacement (ALH), distance average path (DAP), linearity (LIN; VSL/VCL).

\section{Analysis of sperm viability}

All fluorescent assays were performed after $72-96 \mathrm{~h}$ of cooling-storage of sperm. Sperm membrane integrity was evaluated by fluorescently labelled lectin PNA-Alexa Fluor (peanut agglutinin; Molecular Probes, Lucerne, Switzerland) in combination with propidium iodide (PI), which detected dead or necrotic sperm in samples (red signal) and DAPI - blue fluorescent DNA stain (H-1200, Vector Laboratories Inc., Burlingame, CA, USA), which marked all sperm cells in samples. Sperm samples were not fixed, therefore, PNA marked only sperm with disturbed plasma membrane (green signal) whilst sperm with intact membranes remained unstained. Sperm samples were prepared as described before by Makarevich et al. (2011). Staining solution contained $20 \mu \mathrm{mol} \cdot \mathrm{l}^{-1}$ of PNA-Alexa Fluor and $5 \mu \mathrm{g} \cdot \mathrm{ml}^{-1}$ of PI in saline-FCS solution. After $20 \mathrm{~min}$, samples were examined under a Leica fluorescent microscope (MIKRO spol. s r.o., Bratislava, Slovak Republic) with filters for green, red, and blue fluorescence. Sperm with disturbed plasma membrane showed green signal in the acrosomal part of the head.

Apoptotic sperm were detected using specific nuclear green fluorochrome, Yo-Pro-1 (Molecular Probes, Lucerne, Switzerland) in combination with PI. Following washing in a saline-FCS solution, sperm samples were incubated for $20 \mathrm{~min}$ at room temperature in the staining solution containing $5 \mu \mathrm{mol} \cdot \mathrm{l}^{-1}$ of Yo-Pro-1 and $5 \mu \mathrm{g} \cdot \mathrm{ml}^{-1}$ of PI in $200 \mu \mathrm{l}$ of saline-FCS solution. Sperm cells with bright green fluorescent signal were regarded as apoptotic.

Sperm membrane phosphatidylserine (PS) translocation (membrane destabilization) was detected using fluorescently labelled Annexin-V (Roche Slovakia Ltd, Bratislava, Slovak Republic). Sperm samples were prepared as described before by Makarevich et al. (2011). The sperm with PS translocation exhibited green fluorescence and was regarded as sperm with membrane destabilization. Presence of annexin V-positivity in sperm was localized on the acrosomal part of the sperm head, post-acrosomal segment, equatorial segment and on sperm membrane along the entire head (Makarevich et al. 2011).

\section{Statistical analysis}

The experiments were done in four replications. In each experimental group, at least 8 view fields were evaluated (more than 800 sperm cells in each group were analyzed). For sperm motility analysis (CASA), 7 view fields in each experimental group were evaluated, so that at least 500 sperm cells per one experimental group were counted. Mean values were calculated from three measurements $(0 \mathrm{~h}, 0.5 \mathrm{~h}, 2 \mathrm{~h})$ during the day. The results of fluorescent and motility analysis were statistically evaluated by two-way ANOVA test $(P<0.05)$ and graphically processed using SigmaPlot graphic software (version 9.0).

\section{Sperm motility}

\section{Results}

For sperm motility analyses, each sample was analyzed at the time intervals of $0 \mathrm{~h}$, $0.5 \mathrm{~h}$ and $2 \mathrm{~h}$, every day during $72 \mathrm{~h}$ of cooling storage. Caffeine positively affected sperm total motility immediately after its addition (day 0 ); the greatest effect was visible at the dose of $4 \mathrm{mmol}^{-1} \mathrm{l}^{-1}$. Sperm motility was the highest after $0.5 \mathrm{~h}$ of caffeine exposure, when all three concentrations significantly increased sperm motility. Following 24 and $48 \mathrm{~h}$ of sperm cooling storage, caffeine significantly $(P<0.05)$ increased sperm total motility at concentration of 1 and $4 \mathrm{mmol}^{-1} \mathrm{l}^{-1}$ (Fig. 1). Following the next $72 \mathrm{~h}$ of cooling storage, caffeine at all three concentrations significantly $(P<0.05)$ increased sperm motility after addition $(0 \mathrm{~h})$ and after $0.5 \mathrm{~h}$. After $2 \mathrm{~h}$ of incubation at $37^{\circ} \mathrm{C}$, sperm motility extremely decreased in 


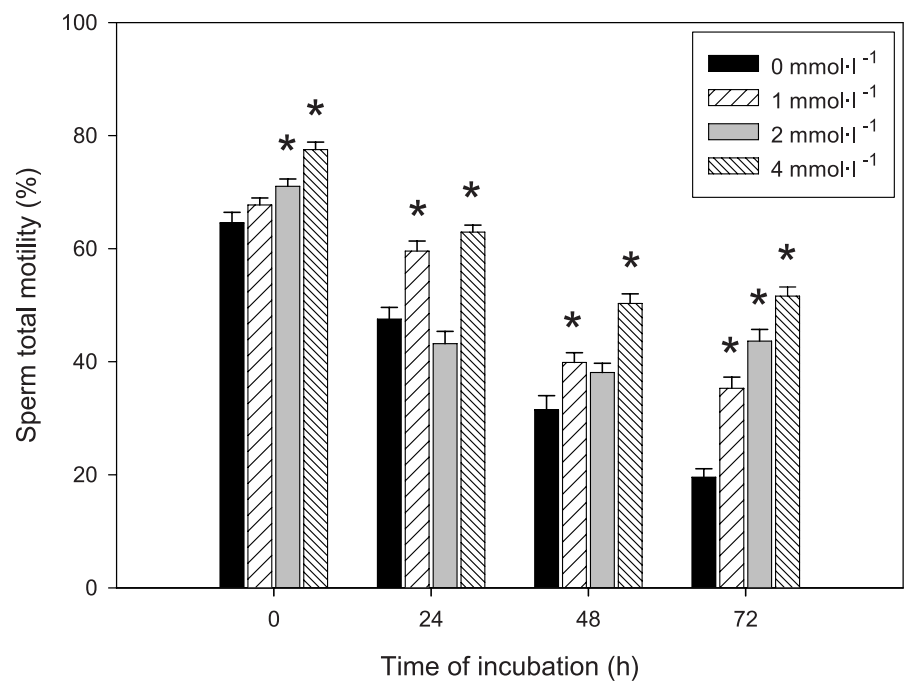

Fig. 1. Effect of caffeine on sperm total motility after cooling storage *Significant difference $(P<0.05)$ compared to control $\left(0 \mathrm{mmol} \cdot \mathrm{l}^{-1}\right)$

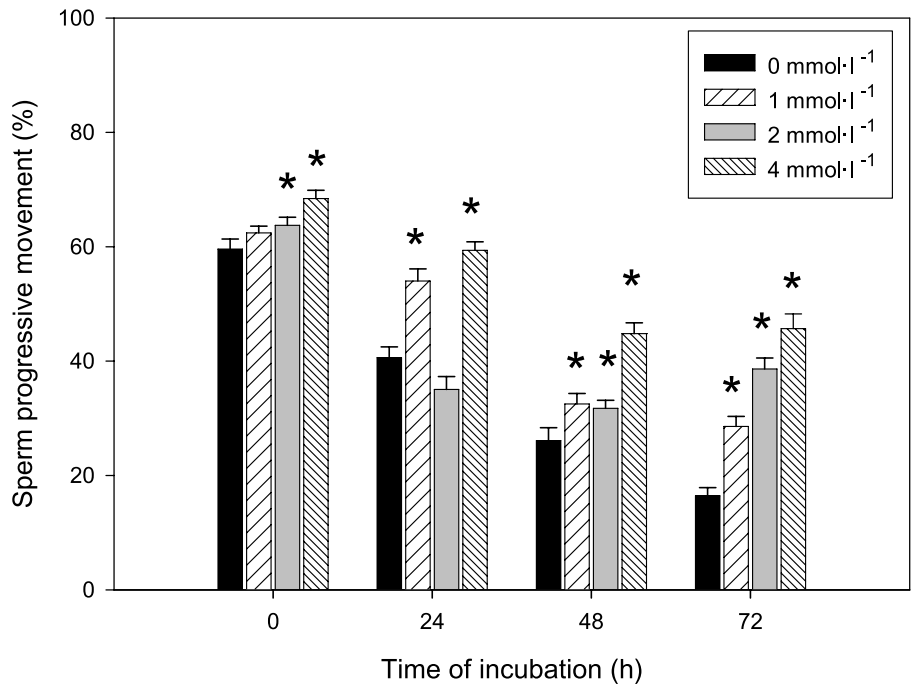

Fig. 1. Effect of caffeine on sperm progressive movement after cooling storage

*Significant difference $(P<0.05)$ compared to control $\left(0 \mathrm{mmol}^{-1} \mathrm{l}^{-1}\right)$

both control and experimental groups; none of the tested caffeine concentrations positively affected this indicator.

Similarly, caffeine at the concentration of 2 and $4 \mathrm{mmol} \cdot \mathrm{l}^{-1}$ positively affected sperm progressive movement on day 0 compared to the control group. After $24 \mathrm{~h}$ of cooling 
storage, caffeine (at the concentration of 1 and $4 \mathrm{mmol} \cdot \mathrm{l}^{-1}$ ) positively affected progressive movement of ram sperm measured at all time points $(0 \mathrm{~h}, 0.5$ and $2 \mathrm{~h})$. Caffeine at the concentration of $2 \mathrm{mmol} \cdot \mathrm{l}^{-1}$ did not alter indicators of motility. The positive effect of caffeine on PM after $48 \mathrm{~h}$ of cooling storage was shown at all tested concentrations. More expressed effect was observed at the highest caffeine concentration $\left(4 \mathrm{mmol} \cdot \mathrm{l}^{-1}\right)$, where PM increased from $26.1 \%$ (control group) to $44.8 \%$ (Fig. 2). After $72 \mathrm{~h}$ of cooling storage, PM in the control group was $16.45 \%$; however, immediately after caffeine addition, all tested concentrations significantly $(P<0.05)$ increased the ratio of progressively moving spermatozoa. The highest effect was visible at the concentration of $4 \mathrm{mmol}^{-1}(45.7 \%)$ (Fig. 2). Caffeine at the concentration of 1 and $4 \mathrm{mmol} \cdot \mathrm{l}^{-1}$ insignificantly increased some of the velocity variables (VAP, VCL) after $24 \mathrm{~h}$ of sperm cooling storage (Table 1). There was a tendency to slight decrease in VAP and VCL at the concentration of $2 \mathrm{mmol} \cdot \mathrm{l}^{-1}$ of caffeine compared to control, whilst other motility indicators were not affected.

Table 1. The effect of caffeine on selected sperm motility characteristics following $24 \mathrm{~h}$ of incubation under cooling conditions.

\begin{tabular}{lcccc}
\hline & \multicolumn{4}{c}{ Concentration of caffeine $\left(\mathrm{mmol} \cdot \mathrm{l}^{-1}\right)$} \\
\cline { 2 - 5 } Indicator & 0 & 1 & 2 & 4 \\
\hline Motility $(\%)$ & $47.55 \pm 2.06$ & $59.58 \pm 1.77^{*}$ & $43.21 \pm 2.16$ & $62.93 \pm 1.24^{*}$ \\
PM $(\%)$ & $40.60 \pm 1.89$ & $54.01 \pm 2.11^{*}$ & $35.04 \pm 2.25$ & $59.38 \pm 1.48^{*}$ \\
ALH $(\mu \mathrm{m})$ & $4.52 \pm 0.13$ & $4.6 \pm 0.1$ & $3.74 \pm 0.13$ & $3.81 \pm 0.09$ \\
DAP $(\mu \mathrm{m})$ & $35.71 \pm 1.02$ & $37.2 \pm 0.89$ & $28.51 \pm 1.29$ & $39.25 \pm 0.58$ \\
VCL $(\mu \mathrm{m} / \mathrm{s})$ & $154.94 \pm 4.90$ & $157.89 \pm 4.47$ & $119.32 \pm 4.98$ & $146.76 \pm 2.70$ \\
VSL $(\mu \mathrm{m} / \mathrm{s})$ & $64.32 \pm 1.97$ & $68.23 \pm 1.6$ & $51.43 \pm 2.85$ & $75.98 \pm 1.41$ \\
VAP $(\mu \mathrm{m} / \mathrm{s})$ & $79.70 \pm 1.94$ & $83.03 \pm 2.11$ & $63.26 \pm 2.72$ & $86.57 \pm 1.61$ \\
LIN $(\mathrm{VSL} / \mathrm{VCL})$ & $0.43 \pm 0.01$ & $0.45 \pm 0.01$ & $0.43 \pm 0.01$ & $0.52 \pm 0.01$ \\
\hline
\end{tabular}

PM - progressive motility, ALH - amplitude of lateral head displacement, DAP - distance average path, VCL curvilinear velocity, VSL - straight line velocity, VAP - average path velocity, LIN - linearity. Data are expressed as mean \pm standard error, ${ }^{*}$ significant difference $(P<0.05)$ compared to control $\left(0 \mathrm{mmol} \cdot \mathrm{l}^{-1}\right)$

\section{Sperm viability}

Neither of the tested caffeine concentrations significantly affected sperm plasma membrane integrity, which was expressed as the proportion of PNA-positive sperm. Caffeine at the concentration of 2 or $4 \mathrm{mmol} \cdot \mathrm{l}^{-1}$ significantly $(P<0.05)$ reduced the proportion of dead/ necrotic sperm in the sample; at the highest concentration $\left(4 \mathrm{mmol} \cdot \mathrm{l}^{-1}\right)$ caffeine significantly $(P<0.05)$ reduced the proportion of apoptotic sperm. Caffeine at the concentration of 1 and $4 \mathrm{mmol} \cdot \mathrm{l}^{-1}$ reduced the percentage of sperm with membrane destabilization (membrane PS translocation) (Table 2). The most of annexin-V positive sperm were labelled in the acrosomal part of the head independent of caffeine concentration.

\section{Discussion}

Caffeine is able to increase the cellular cyclic AMP level, motility and respiration in epididymal and ejaculated bovine spermatozoa (Martinez et al. 2012). The cAMP analogue Sp-cAMP, is a phosphodiesterase inhibitor controlling the intracellular c-AMP levels which in turn influences sperm motility (Agarwal and Loughlin 1992). Motility of spermatozoa is among the most important indicators in semen quality assessment. CASA system is widely used for animal sperm motility analysis (Kozdrowski et al. 2007). Our 
Table 2. The effect of caffeine on ram sperm viability indicators following $24 \mathrm{~h}$ of incubation under cooling conditions.

\begin{tabular}{lcccc}
\hline $\begin{array}{l}\text { Caffeine } \\
\text { mmol- } \text { - }^{-1}\end{array}$ & PNA (\%) & Yo-Pro-1 (\%) & PI (\%) & Annexin-V (\%) \\
\hline 0 (control) & $20.31 \pm 2.14$ & $9.50 \pm 1.42$ & $8.35 \pm 1.06$ & $8.77 \pm 1.35$ \\
1 & $21.95 \pm 2.12$ & $10.14 \pm 0.75$ & $8.79 \pm 1.13$ & $6.13 \pm 0.81^{*}$ \\
2 & $23.00 \pm 1.07$ & $8.09 \pm 1.05$ & $6.25 \pm 0.81^{*}$ & $6.48 \pm 0.94$ \\
4 & $21.00 \pm 0.75$ & $5.65 \pm 0.75^{*}$ & $6.70 \pm 0.62$ & $5.08 \pm 0.87^{*}$ \\
\hline
\end{tabular}

PNA - sperm with disturbed plasma membrane, Yo-Pro-1 - apoptotic sperm, PI - dead/necrotic sperm, Annexin-V - sperm with membrane PS translocation. Data are expressed as mean \pm standard error, $*$ significant difference $(P<0.05)$ compared to control $\left(0 \mathrm{mmol} \cdot \mathrm{l}^{-1}\right)$

study confirmed the positive dose-dependent effect of caffeine on sperm total motility. The most marked effect was observed at the highest concentration of $4 \mathrm{mmol} \cdot \mathrm{l}^{-1}$ which positively affected sperm motility at all time points $(0 \mathrm{~h}, 24 \mathrm{~h}, 48 \mathrm{~h}$ and $72 \mathrm{~h})$. Caffeine at a higher concentration $\left(5 \mathrm{mmol}^{-1} \mathrm{l}^{-1}\right)$ may adversely affect the motility of bull sperm (Momozawa and Fuduka 2003). Caffeine concentration of $4 \mathrm{mmol}^{-l^{-1}}$ may probably be the threshold value for stimulating ram sperm. Compared to fresh semen, cooled ram semen suffers from a decrease in motility and alteration of morphology, accompanied by the decline of survival ability in the female reproductive tract, reduction in fertility, and increased embryonic loss (Aisen et al. 2002; Gil et al. 2003). The quality of sperm movement dropped extremely in cold liquid ram semen stored for 3 to 5 days (O'Hara et al. 2010). In our study, caffeine addition had an immediate positive effect on sperm motility and may help to maintain sperm total motility and progressive movement for a longer time $(72 \mathrm{~h})$. Caffeine at the concentration of $2 \mathrm{mmol} \cdot \mathrm{l}^{-1}$ did not have either positive or negative impact on total motility or progressive movement after $24 \mathrm{~h}$ of cooling storage, although at concentrations of 1 or $4 \mathrm{mmol} \cdot \mathrm{l}^{-1}$ these indicators improved. This discrepancy may be explained by the possible bi-phasic effect of caffeine. Motility in the control group was relatively high after $24 \mathrm{~h}$ of incubation, and so the stimulating effect of caffeine at $2 \mathrm{mmol} \cdot \mathrm{l}^{-1}$ was not expressed. After 48 and $72 \mathrm{~h}$ of sperm cooling storage, motility in the control group decreased markedly, and the stimulating effect of caffeine was well-expressed. All the tested caffeine concentrations significantly increased sperm progressive movement after $48 \mathrm{~h}$ and $72 \mathrm{~h}$ of cooling storage. Except for total motility and progressive movement, there are other indicators of sperm movement characteristic of its quality, such as the ALH, VCL and LIN. In our study, caffeine at the dose of $4 \mathrm{mmol} \cdot \mathrm{l}^{-1}$ moderately increased VSL, VAP and LIN (but not ALH) after $24 \mathrm{~h}$ of sperm cooling storage. It is likely that caffeine, given at the tested doses, increased TM and PM without affecting other indicators of sperm movement. Similarly, caffeine did not improve VAP, VCL, VSL of frozen-thawed equine sperm (Stephens et al. 2013).

There are insufficient data on the effect of caffeine on sperm membrane integrity, occurrence of dead/necrotic and apoptotic sperm in cooling-stored semen. Our results using fluorescein-conjugated lectin PNA-FITC showed that caffeine has no effect on the occurrence of sperm with disturbed plasma membrane in cooled semen. Similarly, Maxwell et al. (1995) reported no effect of caffeine on the acrosome integrity of frozenthawed ram spermatozoa. For successful fertilization, high incidence of sperm with intact membrane is necessary, because intact plasma membrane is an essential requirement for sperm cell function (Makarevich et al. 2011). Spermatozoa with damaged or inactive membranes have limited viability and fertilization potential (Correa and Zavos 1994). 
Annexin $\mathrm{V}$ conjugated to fluorescein serves as a sensitive probe for identification of sperm with loss of membrane asymmetry (Anzar et al. 2002). In our study, all the tested concentrations reduced the percentage of sperm with membrane PS translocation, compared to the control group. Results with human sperm suggest that translocation of PS on the outer leaflet of plasma membrane is mainly related to the acrosome reaction, rather than to apoptosis (Martin et al. 2005). Similarly in our study, the most of annexin-V positive spermatozoa were marked in the acrosomal part of the sperm head, where the acrosome reaction occurs. Presence of apoptotic spermatozoa in fresh semen could be one of the reasons for poor fertility of breeding bulls (Anzar et al. 2002). Our results showed that caffeine at the concentration of $2 \mathrm{mmol} \cdot \mathrm{l}^{-1}$ had no effect, whilst at a higher concentration $\left(4 \mathrm{mmol} \cdot \mathrm{l}^{-1}\right)$ it reduced the occurrence of apoptotic sperm in cooled ram semen. This indicates the concentration-dependent effect of caffeine. Similarly, caffeine at $2 \mathrm{mmol} \cdot \mathrm{l}^{-1}$ decreased the ratio of dead/necrotic sperm in the sample. It is proved that the positive effect of inhibiting phosphodiesterase activity may result in an increase in intracellular cAMP (Funahashi and Nagai 2001) which can influence the incidence of apoptotic cells. There are evidences that cAMP prevented spontaneous apoptosis in ovarian follicles (Flaws et al. 1995). It is possible that caffeine-induced increase in cAMP can lead to a decrease of apoptotic sperm number.

Our results indicate that caffeine has a positive impact on cooling-stored sperm motility and is able to maintain sperm motility for a longer time. Simultaneously, caffeine may reduce the occurrence of sperm with membrane PS translocation or dead/necrotic and apoptotic sperm.

\section{Acknowledgement}

The study was supported by the Slovak Research and Development Agency (SRDA) grants APVV-0514-07 and APVV- 0556-011; and by the Slovak Ministry of Education KEGA No. 012 UPJŠ-4/2011.

\section{References}

Agarwal A, Loughlin KR 1992: Effect of Sp-cAMP on sperm motility in patients with unexplained infertility. Andrologia 24: 53-55

Aisen EG, Medina VH, Venturino A 2002: Cryopreservation and post-thawed fertility of ram semen frozen in different trehalose concentrations. Theriogenology 57: 1801-1808

Anzar M, He L, Buhr MM 2002: Sperm apoptosis in fresh and cryopreserved bull semen detected by flow cytometry and its relationship with fertility. Biol Reprod 66: 354-360

Colas C, Cebrian-Pérez JA, Muino-Blanco T 2010: Caffeine induces ram sperm hyperactivation independent of cAMP-dependent protein kinase. Int J Androl 33: 187-197

Correa JR, Zavos PM 1994: The hypoosmotic swelling test: Its employment as an assay to evaluate the functional integrity of the frozen-thawed bovine sperm membrane. Theriogenology 42: 351-360

Flaws JA, DeSanti A, Tilly KI, Javid RO, Kugu K 1995: Vasoactive intestinal peptide-mediated suppression of apoptosis in the ovary: potential mechanisms of action and evidence of a conserved antiatretogenic role through evolution. Endocrinol 136: 4351-4359

Fraser LR, Monks N 1990: Cyclicnucleotides and mammalian sperm capacitation. J Reprod Fertil 42: 9-21

Funahashi H, Nagai T 2001: Regulation of in vitro penetration of frozen-thawed boar spermatozoa by caffeine and adenosine. Molec Reprod Dev 58: 424-431

Gil J, Lundeheim N, Serquist L, Rodriguez-Martinez H 2003: Influence of extender, temperature, and addition of glycerol on post-thaw sperm parameters in ram semen. Theriogenology 59: 1241-1255

Kozdrowski R, Dubiel A, Bielas W, Dieciol M 2007: Two protocols of cryopreservation of goat semen with the use of computer-assisted semen analysis system. Acta Vet Brno 76: 601-604

Makarevich AV, Spalekova E, Olexikova L, Lukac N, Kubovicova E, Hegedusova Z 2011: Functional characteristics of ram cooling-stored spermatozoa under the influence of epidermal growth factor. Gen Physiol Biophys 30: $36-43$

Martin G, Sabido O, Durand P, Levy R 2005: Phosphatidylserine externalization in human sperm induced by calcium ionophore A23187: relationship with apoptosis, membrane scrambling and the acrosome reaction. Hum Reprod 20: 3459-3468

Martinez AS, Camacho JH, Correa JS 2012: Motility and functional state of the membrane of caprine capacitated spermatozoa under different chemical agents. Open J Vet Med 2: 98-103 
Maxwell WM, Robinson SJ, Roca J, Molinia FC, Sanchez-Partida LG, Evans G 1995: Motility, acrosome integrity and fertility of frozen ram spermatozoa treated with caffeine, pentoxifylline, cAMP, 2-deoxyadenosine and kallikrein. Reprod Fertil 7: 1082-1087

Momozawa K, Fuduka Y 2003: Caffeine in fertilization medium is not essential for bovine IVF by fully capacitated spermatozoa. J Reprod Develop 49: 507-512

O'Hara L, Hanrahan JP, Richardson L 2010: Effect of storage duration, storage temperature, and diluent on the viability and fertility of fresh ram sperm. Theriogenology 73: 541-549

Salem M, Mekkawy M, Ahmed N 1992: Effect of cyclic AMP on fructose utilization, progressive motility and protein synthesis by ram spermatozoa. Theriogenology 37: 1061-1074

Singh P, Raina VS 2000: Effect of caffeine, cAMP and cattle seminal plasma on freezability of buffalo bull semen. Asian-Aust J Anim Sci 13: 901-905

Stephens T, Brooks R, Cheng L, Porr Ch. Splan R 2013: Effects of pentoxifylline, caffeine, and taurine on postthaw motility and longevity of equine frozen semen. J Equine Vet Sci 33: 615-621 